\title{
The effects of protein supplementation during energy balance and energy restriction on whole body protein turnover and metabolism
}

Whole body protein turnover (WBPT) and protein oxidation are metabolically energy costly processes ${ }^{(1)}$ and may contribute to the increased resting and postprandial energy expenditure (EE) on high protein diets ${ }^{(2-4)}$. Additionally, whole body nitrogen and protein balance are essential for maintenance of lean tissue. This study aimed to 1) determine the effects of protein supplementation during energy balance on WBPT and EE, and 2) assess whether protein supplementation during energy restriction (ER) could inhibit the normal downregulation of WBPT and EE commonly induced by energy deficit.

Sixteen participants $(9 \mathrm{M}, 7 \mathrm{~F})$ undertook $4 \times 2$ week study phases; habitual diet 1 (HD1) (subjects consumed their own habitual diet), habitual diet 2 (HD2) (as per HD1), high protein energy balance (HPEbal) $(0.5 \mathrm{~g} / \mathrm{kg} / \mathrm{d}$ whey protein supplement, maintaining energy balance) and high protein energy restriction (HPER) (33\% ER with $0.5 \mathrm{~g} / \mathrm{kg} / \mathrm{d}$ protein supplement, protein intake equal to HPEbal). WBPT (end product method, single dose ${ }^{15} \mathrm{~N}$ glycine, measured in the fed state ${ }^{(5)}$ ), $24 \mathrm{hr}$ nitrogen balance, resting and shortterm postprandial EE (30-40 and 80-90 $\mathrm{min}$ ) were measured at the end of each diet phase.

WBPT kinetics during the study are shown in Fig 1. HPEbal resulted in significant increases in protein flux and balance. HPER resulted in significant increases in protein synthesis however also increases in breakdown. Protein balance during HPER remained significantly higher than habitual diet and participants maintained a positive nitrogen balance (data not shown). There was no effect of HPEbal or HPER on REE either unadjusted or adjusted for changes in FM and FFM (Fig 2). Postprandial EE above REE was not different between study phases though was higher after adjusting for energy intake during HPEbal and HPER (Fig 3). Positive correlations were found between protein balance and REE during HPER $(r=0.720, P<0.01)$ and between protein oxidation with postprandial EE during HD1 $(r>0.536, P<0.05)$ and HPEbal $(r>0.738, P<0.01)$. However there were no other relationships between protein metabolism and EE.

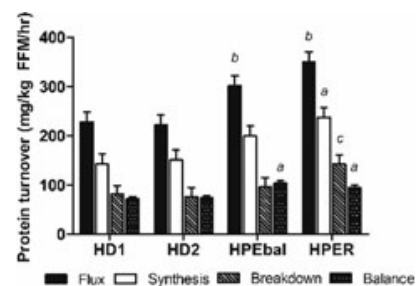

Fig. 1. WBPT Kinetics. Mean (SEM) Significantly greater than HD1 and HD2 $a(\mathrm{P}<0.0001), b(P<0.01), c(P<0.05)$.

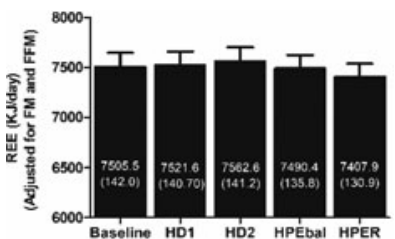

Fig. 2. REE across the study. Mean (SEM)

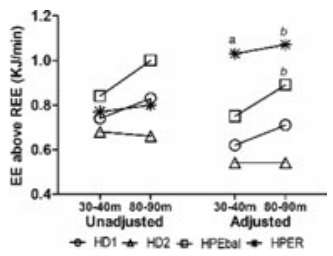

Fig. 3. Postprandial EE above resting at $30-40$ and 80-90 min post meal consumption unadjusted and adjusted for energy intake. $a$ significantly greater than HD1 and HD2. $(P<0.05) b$ significantly greater than $\mathrm{HD} 2(P<0.05)$

These findings support protein supplementation particularly during ER for increasing WBPT and maintaining a positive protein and nitrogen balance. This may assist in the maintenance of lean tissue during ER. Further the results indicate the benefit of protein supplementation for sustaining resting and postprandial EE which were maintained during ER despite an energy deficit and smaller meal sizes. There was no compelling relationships indicating relatively higher rates of WBPT and protein oxidation contribute to higher resting or postprandial energy expenditure.

1. Westerterp-Plantenga MS, Nieuwenhuizen A. Tome D, et al. (2009) Annu Rev Nutr 29(1), 21-41.

2. Mikkelsen PB, Toubro S, Astrup A (2000) 72(5), 1135-1141

3. Robinson SM, Jaccard C, Persaud C, et al. (1990) Am J Clin Nutr 52(1), 72-80.

4. Lejeune MPGM, Westerterp KR, Adam TCM, et al. (2006) Am J Clin Nutr 83(1), 89-94.

5. Grove G, Jackson AA (1995) Br.J.Nutr 74(4), 491-507. 